\section{VBR 38}

THE THERAPEUTIC IMPLICATIONS OF IMMUNOSTIMULATION BY MAFOSFAMIDE

J. Pohl, P. Hilgard, T. Reissmann, J. Stekar and R. Voegeli

Cyclophosphamide, an oxazaphosphorine derivative, stimulates the delayed-type hypersensitivity reaction. This is thought to be due to an elimination of precursors of Tsuppressor cells. In some experimental animal tumour models there is evidence that an elimination of suppressor mechanisms is responsible for the therapeutic efficacy of oxazaphosphorines in low, non-toxic doses. We present results obtained with the myeloid rat leukemia L5222. Treatment of tumour-bearing animals with low doses cyclophosphamide ( $1-20 \mathrm{mg} / \mathrm{kg}$ body weight) resulted in a high proportion of cured animals (survival $>60$ days), higher doses only prolonged the survival time. Very high doses $(100-147 \mathrm{mg} / \mathrm{kg}$ body weight) resulted again in some cures, but further increase of dosage was prohibited by the toxicity of the drug. Similar results, but with a wider immunotherapeutic range, were obtained with mafosfamide, a stable active derivative of 4-hydroxy-cyclophosphamide. The spleens of animals treated with low-dose cyclophosphamide contained transplantable tumour cells for up to five days after treatment, in contrast the spleens of animals treated with high doses were free of transplantable leukemic cells already after one day. Animals $\mathrm{Cu}-$ red with low doses were resistant to reimplantation of the tumour. Similar to non-pretreated control animals, those cured with high doses died from tumour 8-9 days after reimplantation. These results suggest a participation of host defence mechanisms in the curative effect of low-dose oxazaphosphorines on the L5222 leukemia. As mafosfamide needs no enzymatic activation, it is possible to obtain reproducible and predictable plasma levels of the active metabolite, 4-hydroxy-cyclophosphamide. Therefore mafosfamide is the preferable candidate for clinical and preclinical immunopharmacological studies. Abteilung Experimentelle Tumorforschung, Asta-Werke AG, Degussa Pharma Gruppe, D-4800 Bielefeld 14

\section{VBR 39}

PHARMACOKINETICS OF DOXORUBICIN IN PLASMA, TUMOR AND TISSUES IN THE NUDE MOUSE: METHODS AND FIRST RESULTS M.E. ScheuTen, M. Bamberg, V. Budach, T. Heidrich and C.G. Schmidt

Only little is known about the pharmacodynamics of anticancer drugs (G. Powis, Cancer Chemother. Pharmacol. 14:177, 1985). Determinations of the pharmacokinetics of cytotoxic agents in plasma, tumor and tissues in correlation to the dose-dependent antineoplastic effect and toxic side effects are essential for the evaluation of pharmacodynamics. In the clinicat setting, investigations on the tumor kinetics of chemotherapeutics are only feasible in leukemias. Thus, with regard to human solid tumors appropriate preclinical determinations in the nude mouse model are required for the solution of urgent clinical problems such as the evaluation of the effect of drug fractionation or chemosensitizers on antitumor efficacy and toxicity of cytotoxic drugs.

We have treated thymusaplastic nude mice with a subcutaneously transplanted human sarcoma with 6,12 , and $24 \mathrm{mg} / \mathrm{kg}$ doxorubicin intravenously. The kinetics of doxorubicin was determined in plasma, tumor, heart and liver after extraction via $\mathrm{C}-18$ Sep-Pak columns or with chloroform/methanol by separation, identification and quantification of the mother compound and its metabolites by high performance liquid chromatography (HPLC) with fluorometric detection.

The results demonstrate that the doxorubicin concentrations in the tumor are proportional to the dose applied. They are in the range of 1.5 to $6 \mathrm{ng} / \mathrm{mg}$ wet weight up to 24 hours after application. In contrast, the initial concentrations in the heart ( $\max .25 \mathrm{ng} / \mathrm{mg}$ wet weight) are significantly higher. They drop to $2 \mathrm{ng} / \mathrm{mg}$ wet weight during 24 hours post injectionem.

In the nude mouse model it is possible to determine the kinetics of antitumor drugs in human turior heterotransplants and toxicologically critical organs such as heart, kidney, lungs and bone marrow to further evaluate their pharmacodynamics for clinical oncology.

West German Tumor Center, Hufelandstr. 55, D-4.300 Essen 1

\section{VBR 40}

ANTIMUTAGENIC FFFECT OF PSYCHOPHARMACEUTICALS IN RATS WITH INDUCED TUMORS (A MODEL FOR PREVENTION AND TREATMENT OF HUMAN TUMORS)

A.v.Metzler, C. Nitsch

Chromosomal changes play a major role in tumor development. X-rays, carcinogenic substances and chemotherapy are comutagens ( 1 ). We assume that several psychopharmaceutical drugs and neurotransmitters, respectively, have an antimutagenic effect. Rats, bearing 3 -mc sarcomas hardly response to clinical therapeutic measures like chemotherapy, X-rays or surgical tumor removal.

If psychopharmaceuticals were given before tumor appearance, we observed a reduction in tumor rate (2). Given after tumor appearance, survival rate increases threefold and even disappearance of tumors was observed. When treating after surgical removal of tumors, survival rate increased to 97\%, and metastasis could not be demonstrated. Treatment with cylophosphamide alone was ineffective. In combination with psychopharmaceuticals (Piracetam) we achieved tumor remission of $68 \%$ and lessening of toxic side effect of cyclophosphamide. Experimental carcinogenesis - whether by induced or transplanted tumors - effects different functions of the central nervous system. We used the EEG and changes in neurotransmitter metabolism as parameters.

In current clinical studies, psychopharmaceuticals were able to diminish side effects of chemotherapy in children with leucemia.

(1)E.Gebhard, Chem. Carzinogenese 1977

(2) A.v.Metzler and C.Nitsch, Naturwissensch.1985

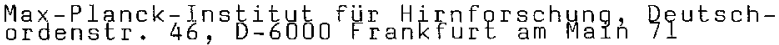

ML 03

MAIN LECTURE ANOREXIA, ABSORPTION, METABOLISM AND NUTRITION IN CANCER DISEASE (CD) E. Holm

I. Pathophysiology of Malnutrition (MN). Body composition in CD with MN differs from that in anorexia nervosa: while muscle and adipose tissue are reduced in both states, visceral organs (heart, liver, kidneys, spleen) contribute to weight loss only in anorexia nervosa and not in $C D$. The mechanisms of cancer-induced anorexia are poorly defined; abnormalities in taste sensation, altered neurotransmitter profiles and learned food aversions may diminish food intake. Malabsorption occurs relatively 1ate. We found amino acid absorption from a peptide diet to be hardly impaired by chemotherapy. Postabsorptive energy expenditure per whole body potassium has been ascertained to be raised in cancer patients. A negative energy balance, mainly accounted for by intensified gluconeogenesis and increased hepatic protein synthesis, appears to be a salient cause of MN. As to the tumor itself, glutamine is a potential energy source. II. Nutritional Therapy (NT). Currently, NT is not specifically directed at the metabolic deviations in $C D$. Nevertheless, dietary measures, supplementary formula preparations, the various tube-feeding procedures and total parenteral nutrition have been shown to restore or maintain body weight, nitrogen balance and body fat. This holds true for nutritional support without antitumor treatment, the indications for such support being controversial. In randomized clinical studies on artificial feeding during chemotherapy or radiation therapy, clearcut nutritional benefits but no significant improvement in treatment tolerance, response or survival could be documented. Surgical patients receiving NT had less postoperative complications than controls. On the whole, NT is efficacious in CD, although the metabolic fate of the substrates is altered.

Dept. of Pathophysiolony, Medical Clinic I Mannheim, University of Heidelberg, Th. Kutzer Ufer, D-6800 Mannheim 